\title{
Pre-HEAT: submillimeter site testing and astronomical spectra from Dome A, Antarctica
}

C. A. Kulesa, C. K. Walker, M. Schein, D. Golish, N. Tothill, et al.

C. A. Kulesa, C. K. Walker, M. Schein, D. Golish, N. Tothill, P. Siegel, S. Weinreb, G. Jones, J. Bardin, K. Jacobs, C. L. Martin, J. Storey, M. Ashley, J. Lawrence, D. Luong-Van, J. Everett, L. Wang, L. Feng, Z. Zhu, J. Yan, J. Yang, X.-G. Zhang, X. Cui, X. Yuan, J. Hu, Z. Xu, Z. Jiang, H. Yang, Y. $\mathrm{Li}, \mathrm{B}$. Sun, W. Qin, Z. Shang, "Pre-HEAT: submillimeter site testing and astronomical spectra from Dome A, Antarctica," Proc. SPIE 7012, Groundbased and Airborne Telescopes II, 701249 (10 July 2008); doi: 10.1117/12.789741

Event: SPIE Astronomical Telescopes + Instrumentation, 2008, Marseille, France 


\title{
Pre-HEAT: submillimeter site testing and astronomical spectra from Dome A, Antarctica
}

C.A. Kulesa ${ }^{\mathrm{a}}$, C.K. Walker ${ }^{\mathrm{a}}$, M. Schein ${ }^{\mathrm{a}}$, D. Golish ${ }^{\mathrm{a}}$, N. Tothill ${ }^{\mathrm{b}}$, P. Siegel ${ }^{\mathrm{c}}$, S. Weinreb ${ }^{\mathrm{c}}$, G. Jones ${ }^{\mathrm{c}}$, J. Bardin $^{\mathrm{c}}$, K. Jacobs ${ }^{\mathrm{d}}$, C.L. Martin ${ }^{\mathrm{e}}$, J. Storey ${ }^{\mathrm{f}}$, M. Ashley ${ }^{\mathrm{f}}$, J. Lawrence ${ }^{\mathrm{f}}$, D. Luong-Van ${ }^{\mathrm{f}}$, J. Everett ${ }^{\mathrm{f}}$, L.

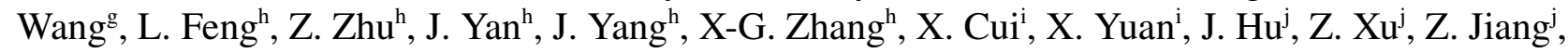
H. Yang ${ }^{\mathrm{k}}$, Y. Li ${ }^{\mathrm{k}}$, B. Sun ${ }^{\mathrm{k}}$, W. Qin ${ }^{\mathrm{k}}$, Z. Shang

${ }^{a}$ University of Arizona, USA

${ }^{b}$ University of Exeter, UK

${ }^{\mathrm{c} C a l i f o r n i a}$ Institute of Technology, USA

${ }^{\mathrm{d}}$ University of Cologne, Germany

${ }^{\circ}$ Oberlin College, USA

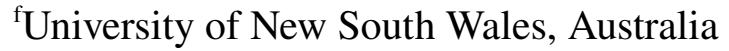

${ }^{\mathrm{g}}$ Texas A\&M University, USA

${ }^{h}$ Purple Mountain Observatory, China

${ }^{i}$ Nanjing Institute of Astronomical Optics Technology, China

${ }^{\mathrm{j} N a t i o n a l ~ A s t r o n o m i c a l ~ O b s e r v a t o r y ~ o f ~ C h i n a, ~ C h i n a ~}$

${ }^{k}$ Polar Research Institute of China, China

'Tianjin Normal University, China

\begin{abstract}
Pre-HEAT is a $20 \mathrm{~cm}$ aperture submillimeter-wave telescope with a $660 \mathrm{GHz}$ (450 micron) Schottky diode heterodyne receiver and digital FFT spectrometer for the Plateau Observatory (PLATO) developed by the University of New South Wales. In January 2008 it was deployed to Dome A, the summit of the Antarctic plateau, as part of a scientific traverse led by the Polar Research Institute of China and the Chinese Academy of Sciences. Dome A may be one of the best sites in the world for ground based Terahertz astronomy, based on the exceptionally cold, dry and stable conditions which prevail there. Pre-HEAT is measuring the 450 micron sky opacity at Dome A and mapping the Galactic Plane in the ${ }^{13} \mathrm{CO} \mathrm{J}=6-5$ line, constituting the first submillimeter measurements from Dome A. It is field-testing many of the key technologies for its namesake -- a successor mission called HEAT: the High Elevation Antarctic Terahertz telescope. Exciting prospects for submillimeter astronomy from Dome A and the status of Pre-HEAT will be presented.
\end{abstract}

Keywords: site testing, Antarctic astronomy, submillimeter instrumentation, terahertz spectroscopy, heterodyne receiver, interstellar medium, star formation 


\section{INTRODUCTION}

It has long been recognized that astronomical observations in the far-infrared (i.e. terahertz and submillimeter) are strongly attenuated by the opacity of atmospheric water vapor, nitrogen, ozone and oxygen. The most appropriate sites for long-wavelength astronomical telescopes have therefore been high, dry mountain sites. Among these, the most prominent are the summit of Mauna Kea in Hawaii, the Chajnantor plain and summit in the high Atacama desert of northern Chile, and the high Antarctic plateau. With the large-scale development of the Chajnantor plain for the Atacama Large Millimeter Array (ALMA), numerous new submillimeter-wave telescopes have arrived in Chile, ranging from small, dedicated telescopes (e.g. the 0.5-meter RLT $^{1}$ and 4-meter NANTEN2 ${ }^{2}$ telescopes) to large-scale observatories (10-meter $\mathrm{ASTE}^{3}, 12$-meter $\mathrm{APEX}^{4}$, and the planned 25-meter $\mathrm{CCAT}^{5}$ reflector).

With significantly colder and drier conditions than both Mauna Kea and the Chilean Atacama desert $^{6}$, the high ice plateau of the Antarctic continent offers tantalizing prospects for astronomical observations at submillimeter wavelengths. With the advent of the 1.7-meter AST/RO telescope at the geographic South Pole, these prospects were quantified $^{7}$. The South Pole site offers very low water vapor content in winter ( $\sim 0.25 \mathrm{~mm}$ precipitable water vapor) superlative atmospheric stability and generally comparable transparency to the ALMA site at Chajnantor below a frequency of $1 \mathrm{THz}$. At higher frequencies, the significant dry air opacity of the South Pole owing to its comparatively low elevation ( $2.8 \mathrm{~km}$ versus $5.1 \mathrm{~km}$ at Chajnantor) was likely to limit the atmospheric transparency, though observations at $1.5 \mathrm{THz}$ were made from the AST/RO telescope ${ }^{8}$ before it was decommissioned to make way for the 10-meter South Pole Telescope (SPT).

The excellent submillimeter conditions obtainable from the South Pole has led to renewed interest in sites higher up on the Antarctic plateau. In 2003, the University of New South Wales installed the Automated Astrophysical Site Testing International Observatory ${ }^{9}$ (AASTINO) at the $3.2 \mathrm{~km}$ elevation summit of Dome $\mathrm{C}$ with a 350 micron tipping radiometer among its complement of instruments. In its first season of observing, it was clearly demonstrated that conditions were both more stable and transparent than at South Pole ${ }^{10}$. Subsequently, expectations for the $4.1 \mathrm{~km}$ high summit of the Antarctic plateau (Dome A) were high ${ }^{11}$.

It was not until 2005, when the first inland traverse to Dome A was achieved by the Polar Research Institute of China (PRIC), that an automated weather station (AWS) was installed at the site. The AWS measurements have shown that the site is colder, drier, calmer, and exhibits much lower atmospheric pressure than the South Pole (e.g. 570 versus 680 mbar) ${ }^{12}$, indicating favorable conditions for observations at terahertz frequencies.

As a part of a 2008 International Polar Year PANDA expedition to Dome A conducted by PRIC and the Chinese Academy of Sciences (CAS), an international team set out to construct a series of experiments to quantify the site conditions at Dome A and perform initial astronomical observations. The resulting PLATeau Observatory (PLATO) ${ }^{13}$ was designed and constructed at the University of New South Wales in Sydney, Australia in 2006-7, culminating in the integration and testing of the science experiments starting in September 2007, the delivery of the observatory to the Chinese expedition in November 2007, and the successful installation of PLATO at Dome A in January 2008. After a $4000 \mathrm{~km}$ oceanic voyage, a $1300 \mathrm{~km}$ overland traverse, and over 120 days of completely unattended operation, PLATO and its subsystems are still operational as of 26 May 2008.

The submillimeter component of the PLATeau Observatory is called "Pre-HEAT". It is a technological prototype for the High Elevation Antarctic Terahertz telescope (HEAT), and is comprised of a Schottky-diode heterodyne receiver operating at a frequency of $660 \mathrm{GHz}$ (450 microns wavelength) coupled to a $20 \mathrm{~cm}$ aperture single-axis telescope and a digital Fast-Fourier-Transform (FFT) spectrometer. 


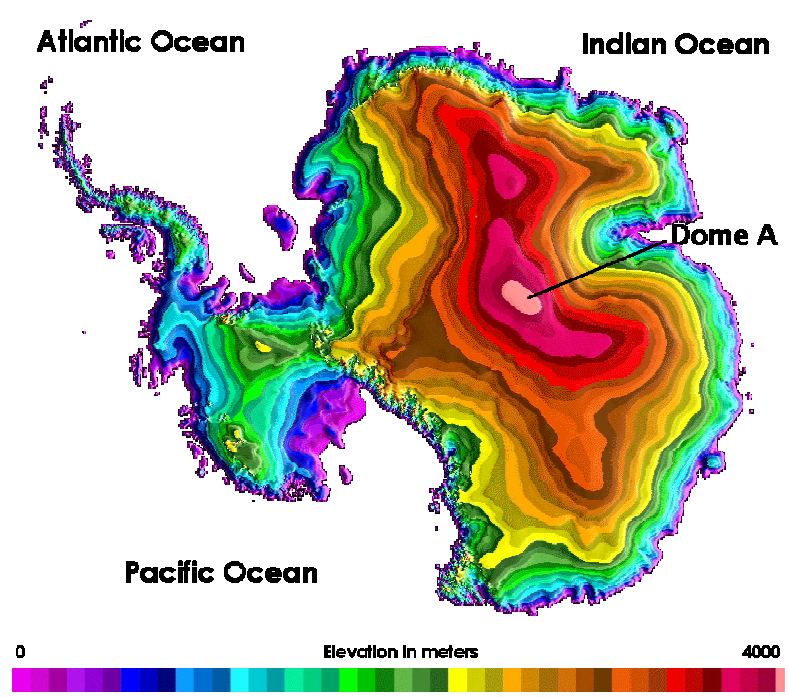

Figure 1: Topographic relief map of the Antarctic plateau,; Dome A represents the summit, near the geographic center of the continent.

\section{DESIGN CONSIDERATIONS}

Pre-HEAT is designed with two principal scientific goals: to measure the 450 micron sky opacity over Dome A, and to perform spectral line observations of the Galactic Plane in the ${ }^{13} \mathrm{CO} \mathrm{J}=6-5$ line at $661 \mathrm{GHz}$. To achieve the same (10 arcminute) angular resolution of the landmark Columbia/CfA Galactic Plane CO J=1-0 surveys ${ }^{14}$, a $20 \mathrm{~cm}$ aperture is required at $660 \mathrm{GHz}$. While pyroelectric detectors are most commonly deployed on submillimeter tipping radiometers, the requirement of sensitive, high-resolution spectroscopy suggests instead the use of an uncooled heterodyne receiver. A Schottky-diode mixer receiver, coupled with commercially-available IF amplifiers and filters ${ }^{15}$, was provided by $\mathrm{P}$. Siegel at JPL/Caltech for the experiment.

PLATO was designed around an average power budget of $\sim 800$ watts during winter, stemming exclusively from a redundant series of six diesel engines in a secondary module separated from the main instrument module by a span of 50 meters. The power specification for Pre-HEAT is therefore $<200$ watts at peak. Since a standard rack-mounted computer alone can absorb this entire power allotment, an embedded systems model permeates the entire design of PreHEAT.

The harshest environmental concern for the design and construction of Pre-HEAT is naturally the extreme cold, from the brightly-lit summers reaching a balmy $-30^{\circ} \mathrm{C}\left(-20^{\circ} \mathrm{F}\right)$ to dark winter nights as low as $-90^{\circ} \mathrm{C}\left(-130^{\circ} \mathrm{F}\right)$. While resistive heaters are available inside the highly insulated PLATO instrument module to provide some temperature stabilization, efficient reuse of instrument waste heat is critical to maintaining the operation of the most sensitive electronics and especially (moving) telescopic components.

Corollary to thermal control is the prevention of frost. Although the cold Antarctic air is only capable of supporting a small burden of water vapor, it is still supersaturated with respect to the frost point of ice. Thus, exposed metal surfaces which couple well to the cold infrared sky will cool radiatively below the frost- and dew-point temperatures, and accumulate ice. Judicious resistive heating of optical surfaces, mechanical deicing measures, and passive insulation and baffling to minimize radiative cooling, are all design requirements for astronomical instrumentation in Antarctica. The additional need for completely autonomous operation for up to a year at a time underlines the overarching need for a modular approach, using simple, robust components with streamlined interfaces. 
Although the operation of PLATO and Pre-HEAT is unmanned, the installation of both would certainly not be! At the high pressure altitude of Dome A (typically $4.5-4.8 \mathrm{~km}$ ), there is little more than $50 \%$ of the sea level oxygen content in the air, and human physical and mental strength deteriorates greatly. Thus, a simple installation procedure with components that can be readily carried by no more than $2-3$ people at altitude is necessary.

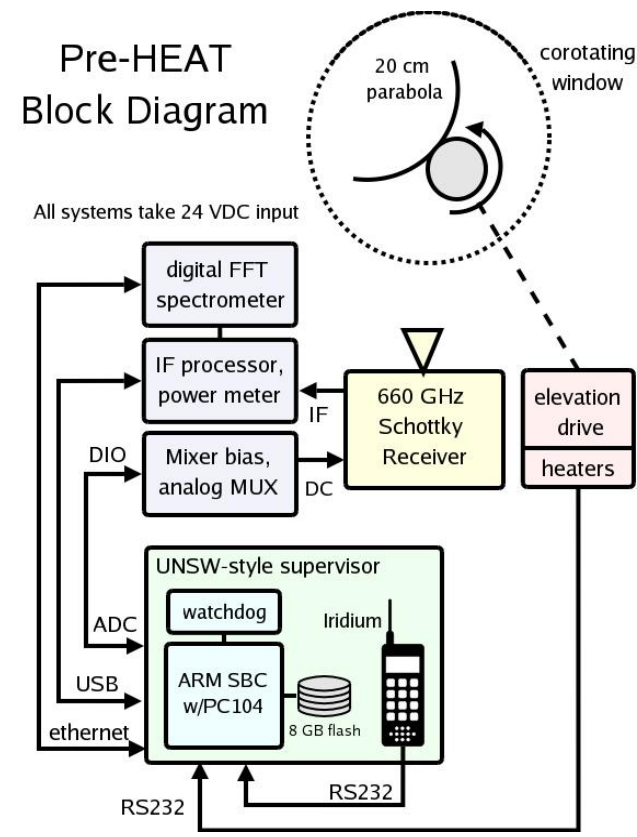

Figure 2: Block diagram of the principal PreHEAT subsystems

\section{PRE-HEAT SUBSYSTEMS}

A block diagram depicting the overall system architecture of the Pre-HEAT experiment is shown in Figure 2. A description of each component follows.

\subsection{Optical and Mechanical Assembly}

The two-piece telescope assembly was designed for ease of installation (Figure 3) and machined at Larson Engineering in Boulder, CO. Two nested aluminum cylinders are spaced by a pair of low-temperature ring bearings from Kaman. The outer cylinder is fitted with a radial mount to match the bolt pattern of the PLATO instrument module, and the rotating inner cylinder houses the telescope. A 1/64" thick sheet of HDPE serves as the entrance window, and a 20x28 $\mathrm{cm} 90^{\circ}$ off-axis $\mathrm{f} / 5$ parabola mounted at one end of the cylinder serves as the primary mirror. The focal length was fixed by the measurement of the Gaussian beam propagating from the feed-horn of the heterodyne receiver, and provided an edge-taper of $10 \mathrm{~dB}$. Standing waves in the tube are reduced by multiple layers of silicon carbide grit embedded in layers of Stycast epoxy, providing an absorptive layer atop the otherwise reflective aluminum surface. Thin aluminum plates (hydrocoptic marzelvanes) baffle the telescope optical beam, reducing the solid angle of cold sky seen by both the primary mirror and window (Figure 4b). Constructed at the University of Exeter, these baffles minimize radiative cooling while allowing convective "heating" from ambient airflow. On the fixed outer tube lies a reference load which the telescope can reference by pointing down toward the ground. This absorptive cell can be heated to two different temperatures to measure the receiver noise temperature and gain for calibration. The fixed reference load is flanked by soft nylon brushes that articulate against the moving cylinder of the telescope structure, removing frost accumulation 
during inclement weather. Such conditions can be detected by careful monitoring of the structure temperature as compared to the ambient air temperature. Thermal sensing of Pre-HEAT is performed by a series of eight AD590 twoterminal IC temperature transducers located throughout the mechanical structure.

Motor control of the telescope is provided by a low-temperature NEMA-34 sized stepper motor from Empire Magnetics, driven by a OEM750X stepper controller from Parker/Compumotor, which is in turn controlled via RS-232 from the Pre-HEAT computer. A cut-away cross section of the telescope assembly is shown in Figure 4a, and a photo of the installed telescope at the summit of Dome A, Antarctica is shown in Figure 4b.

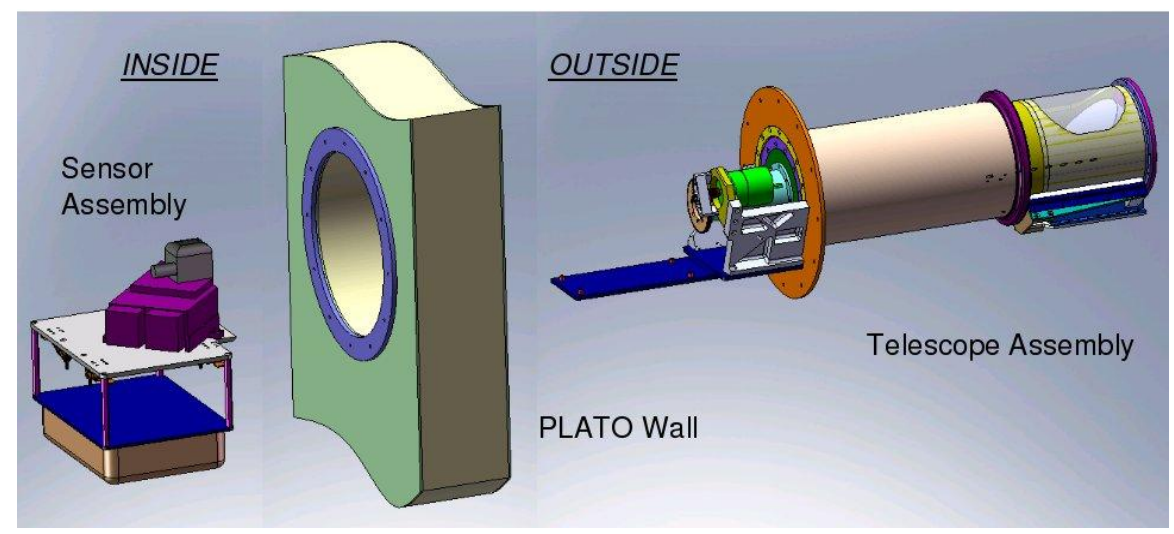

Figure 3: 3D rendering of the designed telescope mechanical structures, demonstrating the ease of assembly on-site. The masses of the inside and outside components are $10 \mathrm{~kg}$ and $35 \mathrm{~kg}$, respectively.

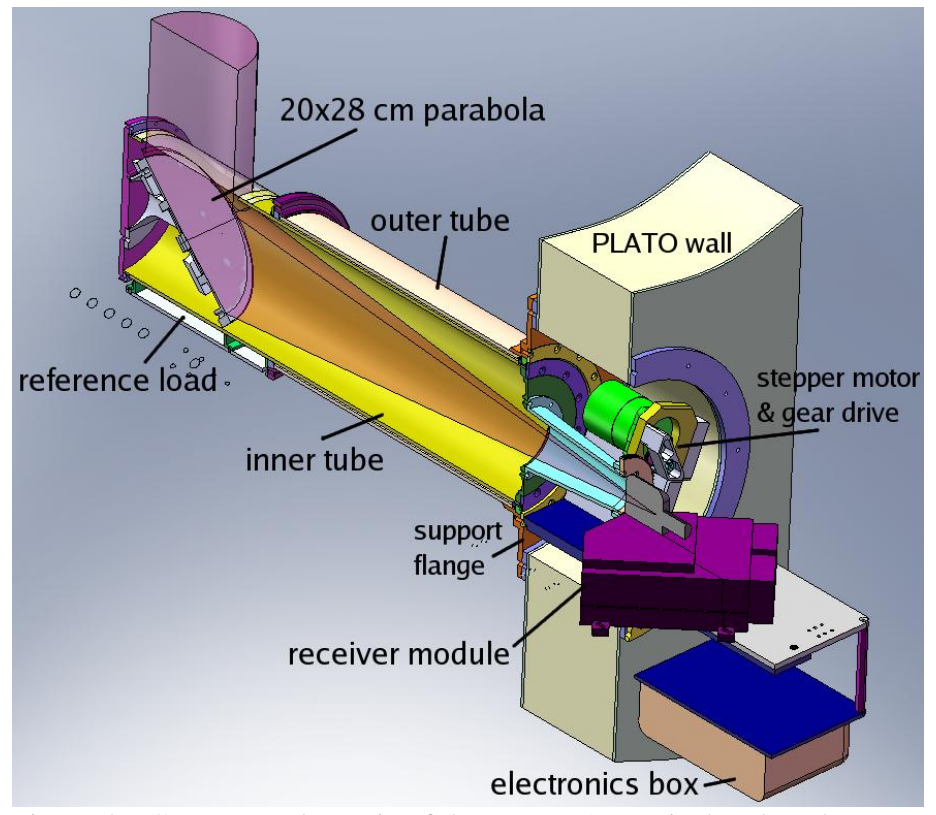

Figure 4a: Cut-away schematic of the Pre-HEAT optical path and mechanical subassemblies.

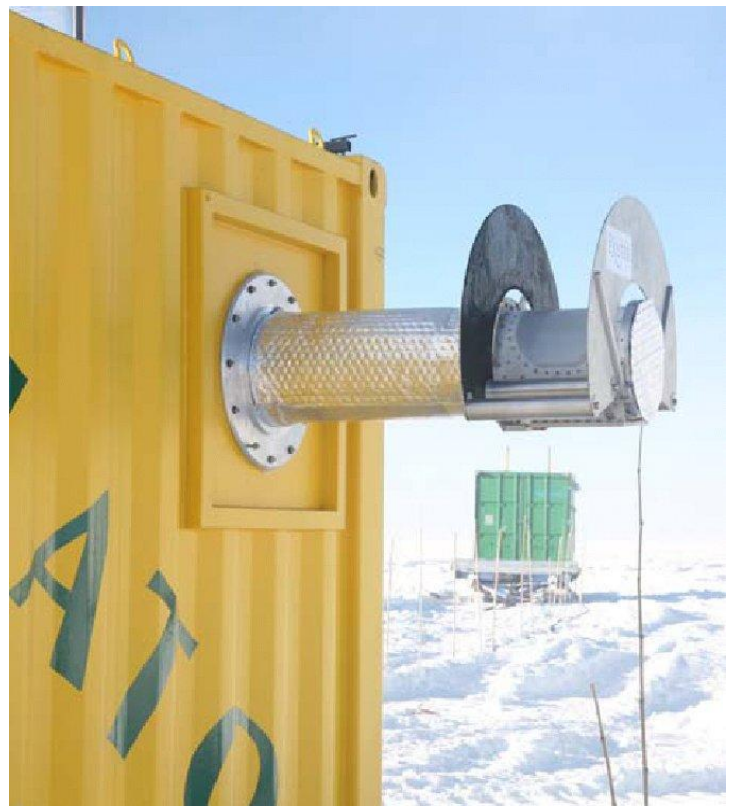

Figure 4b: The Pre-HEAT telescope installed onto the PLATO instrument module at Dome A. Photo from January 2008, courtesy Z. Zhu and Z. Xu. 


\subsection{Power Distribution and Conditioning}

The PLATO-supplied bus voltage is 24-30 VDC, depending on the temperature and charge state of PLATO's 24V, 320 A-hr battery bank. The charge of the 6-gel-cell bank is maintained by diesel generators in winter, and augmented by solar panels in summer. Owing to the diverse needs of the Pre-HEAT electronics, a power conditioning module is needed to distribute proper voltages to the different components of the experiment. A series of efficient (>82\%) switching DC/DC converters are employed to provide separate digital and analog $5 \mathrm{~V},+/-15 \mathrm{~V}$ and $+3.3 \mathrm{~V}$ power buses, as shown in Figure 5. Each DC/DC converter can be remotely controlled using TTL-level logic inputs to fine-tune the power consumption of the instrument, and is bypassed with an appropriate LC "pi" filter for the elimination of switching noise and other transients. Waste heat from the DC/DC converters is heatsunk to the electronics box chassis, coupled to the more sensitive electronics components and thermally strapped to the receiver module to provide sufficient heat to eliminate frost accumulation.

The all-up power consumption of the Pre-HEAT instrument in normal operation is 40 watts, peaking to 90 watts during a telescope slew. External resistive heaters that ward off frost accumulation add an additional 80 watts. Thus, the normal average load presented to PLATO by Pre-HEAT during winter operation is $125-150$ watts.

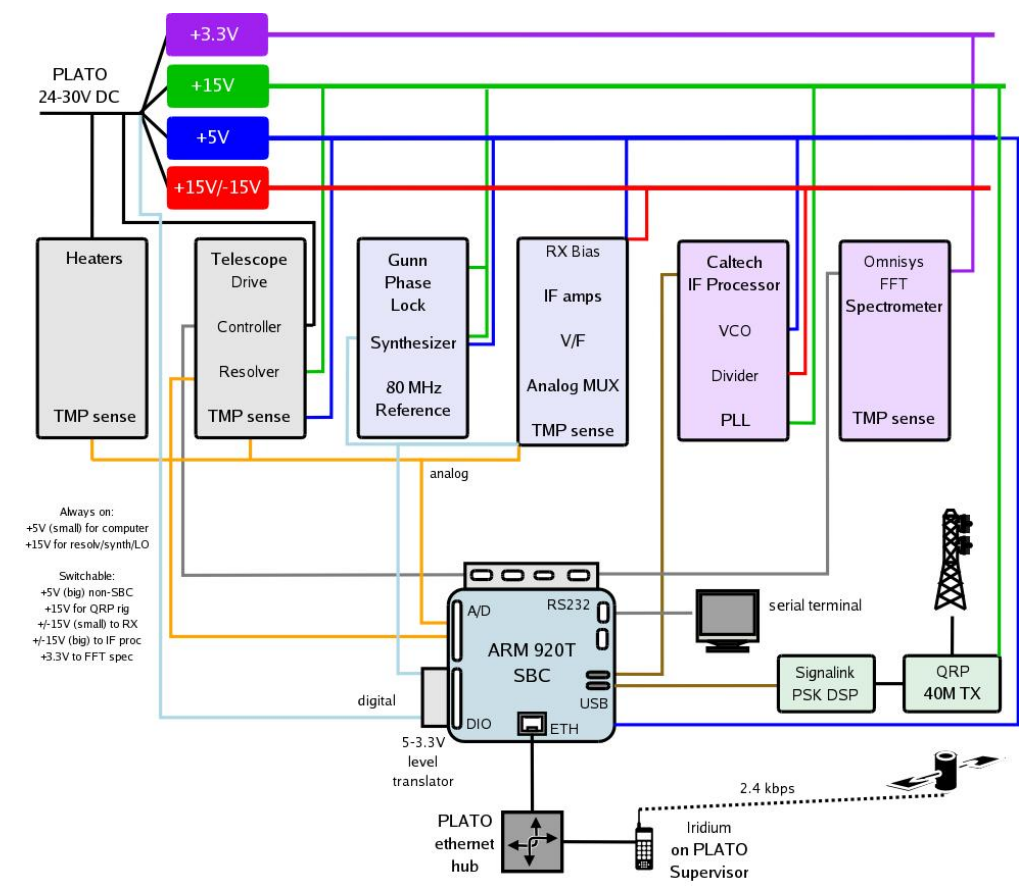

Figure 5: Block diagram of the power distribution module, showing the interrelation of the power buses, the electronics subsystems, and the digital control buses.

\subsection{Receiver Module}

The Pre-HEAT receiver module is derived from the fundamental planar-Schottky-diode mixer receiver provided by P. Siegel at Caltech/JPL, and is shown in Figure 6. The $656 \mathrm{GHz}$ local oscillator (LO) from Radiometer Physics uses an InP Gunn diode oscillator followed by a whisker-contacted Schottky-varactor-diode sextupler multiplier. A folded Fabry-Perot diplexer is used to inject the LO and sky signals into the $660 \mathrm{GHz}$ receiver feed-horn. The measured roomtemperature double-sideband (DSB) noise temperature was $3500 \mathrm{~K}$ at a $5 \mathrm{GHz}$ Intermediate Frequency (IF). At a 
reduced LO power of 250 microwatts (as delivered to Dome A), the noise temperature increased to $\sim 5000 \mathrm{~K}$ DSB.

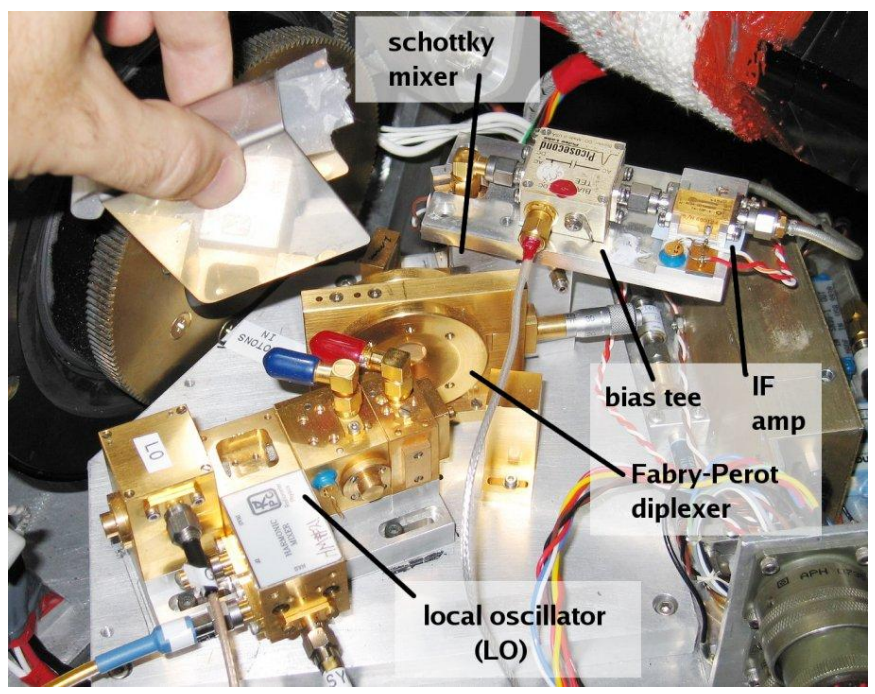

Figure 6: Photo of the Caltech/JPL $660 \mathrm{GHz}$ Schottky-diode mixer receiver as installed onto the Pre-HEAT telescope during integration tests at the University of New South Wales in Sydney.

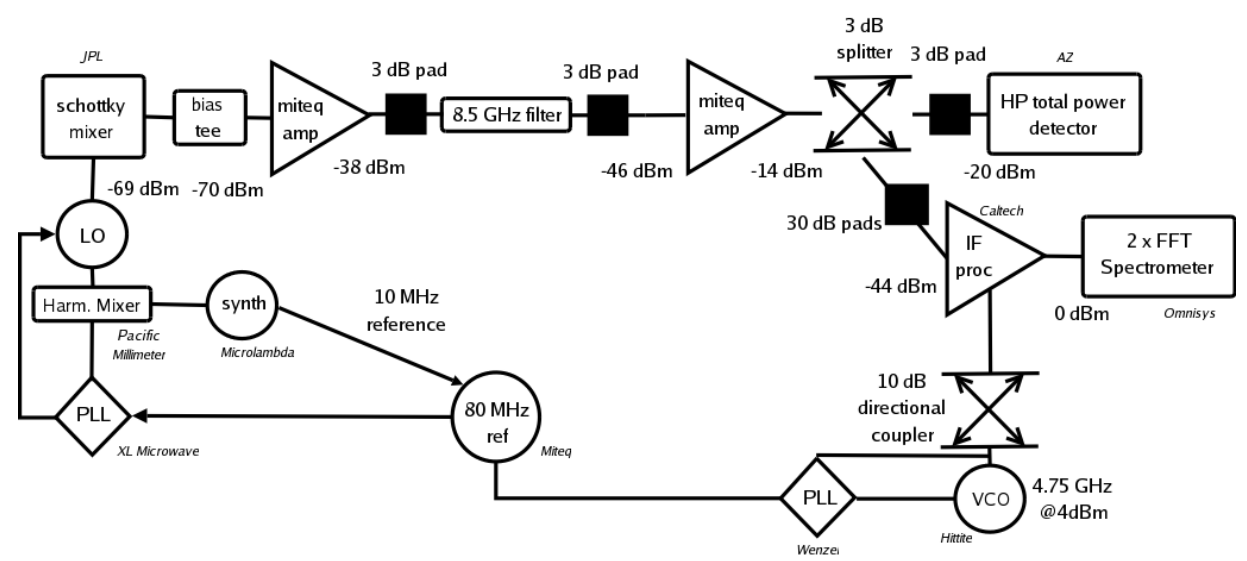

Figure 7: Block diagram of the Pre-HEAT IF processor system

\subsection{IF processor and digital FFT spectrometer}

A block diagram of the Pre-HEAT IF system is shown in Figure 7. The 1-12 GHz wide IF signal from the Schottky mixer is amplified, low-pass filtered, diplexed, and passed to a HP $10 \mathrm{MHz}-18 \mathrm{GHz}$ total power detector, whose analog output is amplified and sent to the Pre-HEAT computer for digitization. The diplexed IF signal is also sent to the spectroscopic IF chain, principally comprised of an IF downconverter and a digital FFT spectrometer. The IF downconverter was built at Caltech as a prototype for the Supercam 64-beam $345 \mathrm{GHz}$ heterodyne array being built at the SORAL lab at the University of Arizona ${ }^{16}$. It mixes the $4.75-5.25 \mathrm{GHz}$ portion of the IF with a phase-locked 4.75 $\mathrm{GHz}$ Hittite local oscillator to provide a 0-500 MHz baseband signal for the FFT spectrometer, after sufficient amplification and analog bandpass filtering to eliminate aliasing from out of band signals. Total power detection is 
provided by a V/F converter followed by a fast counter. Command and control of the IF processor is via a USB interface to an RS232-to-fiber optic transceiver. The FFT spectrometer board (Figure 8) was designed and constructed by Omnisys AB in Sweden and also served as a prototype for the Supercam array. For a total of 10 watts of input DC power, a total of $1 \mathrm{GHz}$ of bandwidth (2 IF inputs) is digitized using two dual-1Gsps Atmel 8-bit ADCs and FFT'd into a power spectrum using an on-board Xilinx Virtex-4 FPGA. For this particular prototype, the output is sent over a serial RS-232 interface to the Pre-HEAT control computer for subsequent processing and storage.

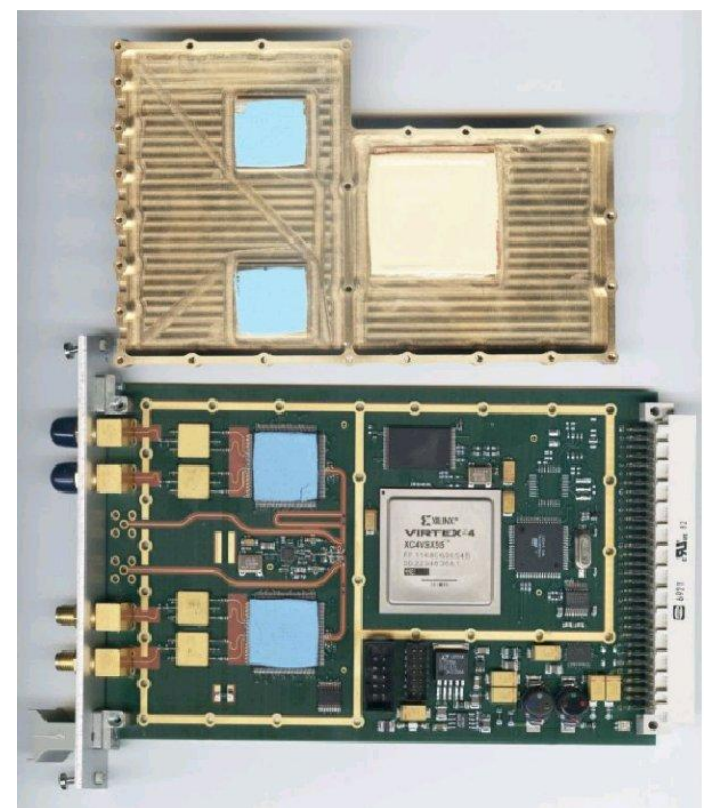

Figure 8: Prototype digital FFT spectrometer board (and machined heatsink) from Omnisys AB provides $1000 \mathrm{MHz}$ of spectrometer bandwidth shared across 2 IF inputs for 10 watts of input DC power.

\subsection{Computer Control and Software}

Robustness of the command and control system for Pre-HEAT is paramount to its success. Based on cost, simplicity of design, cold component testing at $-30^{\circ}$ to $-80^{\circ} \mathrm{C}$, and generality of software design and interface, a TS-7200 single board computer from Technologic Systems was selected for control of Pre-HEAT (Figure 8). A power consumption of 2 watts operates a $200 \mathrm{MHz}$ ARM9 RISC processor with $32 \mathrm{MB}$ of SRAM, PC-104 bus architecture, $100 \mathrm{Mbit}$ ethernet interface, digital I/O, an 8-channel ADC, two USB and two serial (RS-232) interfaces. Mass storage is provided by an industrial-rated CompactFlash card with $8 \mathrm{~GB}$ capacity, rated and tested for operation below $-40^{\circ} \mathrm{C}$. A multi-port serial board and a battery-backed real time clock (RTC) board are added onto the TS-7200's PC-104 bus to augment its capabilities. The NetBSD 4.0 operating system, an open-source derivative of 4.4BSD UNIX, is adopted to operate the TS-7200, owing to the OS's frugal use of resources, rich cross-platform development environment, and simple, robust architecture. The digital I/O lines on the TS-7200 are used to generate the digital (I2C, SPI and synthesizer) buses that course through the electronics box, providing remote control of the receiver and LO bias voltages, phase lock settings, and synthesizer frequency. The many monitored analog signals in Pre-HEAT are routed to an analog multiplexer (MUX) board, which selects one of 32 analog inputs to a single ADC channel on the TS-7200.

Software control of the various Pre-HEAT hardware components (receiver, IF processor, telescope, spectrometer) is modularized into several TCP/IP socket servers, which each listen for ASCII text commands to perform. Observing sequences are scheduled by the UNIX cron daemon and are reconfigured remotely using PLATO's Iridium PPP modem connection using the short burst data (SBD) protocol. Before an observation is carried out, basic environmental and 
instrument configuration checks are performed; if these checks do not pass, an error is reported and the telescope will park at the "stow" position (primary mirror pointed downwards, HDPE window facing the heated reference load) until the next scheduled observation. When "safed", the telescope will still perform periodic short slews to assure that it doesn't freeze in place. This "safe" mode can be overridden by remote software commands.

\section{PRE-HEAT STATUS}

Pre-HEAT was first turned on at the summit of Dome A, Antarctica on 14 January 2008, and achieved first light on 19 January. Manual operation of the instrument proceeded from 24 January until the end of February. Autonomous operation followed in early March and continues to this day (26 May 2008). The performance of the instrument is sufficient to measure the sky opacity at $660 \mathrm{GHz}$, and excellent opacity data has been measured and collected. The total power measurement of the Sun was measured in February, validating the optical performance of the telescope and, together with the Moon, providing pointing calibration sources. It is expected that the first Galactic Plane maps of ${ }^{13} \mathrm{CO}$ $\mathrm{J}=6-5$ are imminent and will be achieved by the end of May. In addition to astronomical detection of ${ }^{13} \mathrm{CO}$, it is also possible to make measurements of atmospheric ozone and other atmospheric trace species, by altering the LO frequency and/or adjusting the 4-5 GHz LO frequency of the Caltech IF downconverter. Such measurements are also imminent, pending minor adjustments to the current suite of software.

Not all has been flawlessly smooth; after a sequence of accidental power failures during the installation of the instruments, the receiver suffered a 4-fold drop in performance. This degradation is consistent with a loss of about half of the local oscillator (LO) power, and is likely at the whisker-contacted schottky-varactor multiplier stages (particularly the final frequency tripler from $219 \mathrm{GHz}$ to $656 \mathrm{GHz}$ ). The receiver has worked stably since, and with the reduced operating temperature $\left(-40^{\circ} \mathrm{C}\right)$ as of the time of this writing, the DSB receiver temperature has improved from its January value of $\sim 16,000 \mathrm{~K}$ to a May value of $\sim 14,000 \mathrm{~K}$. The performance hit has only impacted the rate at which meaningful astronomical spectra can be accumulated, and reduces the mapping coverage of the ${ }^{13} \mathrm{CO}$ survey accordingly.

The (Pre-)HEAT web page can be used to learn more about Pre-HEAT, explore the data taken so far and view graphical displays of preliminary scientific results. The permanent URL is: http://soral.as.arizona.edu/heat/

\section{FUTURE PROSPECTS}

The success of Pre-HEAT and the spectacular conditions at Dome A make the prospects for submillimeter and terahertz observations from the Antarctic plateau very enticing. Indeed, Figure 9 shows the terahertz atmospheric transmission modeled $^{17}$ from Dome A based on the $661 \mathrm{GHz}$ opacity already measured from Dome A, and the conditions are still improving as the Antarctic plateau continues to plunge toward midwinter.

A natural upgrade path from Pre-HEAT, using the same PLATO module, would be the $\sim 50 \mathrm{~cm}$ HEAT telescope. Coupled with 800, 1460, and $1900 \mathrm{GHz}$ subharmonically-pumped, planar-Schottky-diode receivers, HEAT would provide 1-3 arcminute resolution spectral line maps of the Milky Way in the light of the three major forms of carbon in the Galaxy: the $\mathrm{CO}$ molecule in its $\mathrm{J}=7-6$ transition at $806.5 \mathrm{GHz}$, the $\mathrm{J}=2-1$ transition of the fine structure line of atomic carbon, $\mathrm{C}^{0}$, at $809.3 \mathrm{GHz}$, and the ionized carbon $\left(\mathrm{C}^{+}\right)$fine structure line at $1900.5 \mathrm{GHz}$. In addition, the 1461.3 $\mathrm{GHz}$ line of ionized nitrogen $\left(\mathrm{N}^{+}\right)$would provide an extinction-free measure of the low-density warm ionized interstellar medium in the Galaxy, a measure of the star formation rate. In combination with existing $\mathrm{CO}, \mathrm{HI}$ and recombination line maps of the Galaxy, the survey performed by HEAT would help illuminate the complete life cycle of interstellar gas that governs the evolution of galaxies and the formation of star and planet systems. For example, the HEAT surveys should reveal giant molecular clouds forming from diffuse atomic gas for the first time, and determine how much interstellar molecular hydrogen $\left(\mathrm{H}_{2}\right)$ is invisible to conventional millimeter-wave $\mathrm{CO}$ surveys. Such surveys highlight the large-scale ecology of the Galaxy that provides context to the detailed studies of the interstellar medium and star formation that larger facilities like the Stratospheric Observatory for Infrared Astronomy (SOFIA) and the Herschel 
Space Observatory will provide in the near future.

It would be possible to deploy the HEAT telescope in place of the Pre-HEAT telescope as early as the 2009-10 Austral summer. With the more established logistical presence at Dome A that PRIC/CAS intend to create in future years, a cryogenic instrument package for HEAT could be deployed, and naturally, larger submillimeter telescopes could eventually be constructed to take the Galactic science learned from (Pre-)HEAT and apply it as a template for deriving star forming and interstellar properties of distant galaxies. Dome A would also serve as an excellent technological testbed for the development of next-generation (esp. heterodyne) detector arrays before their application to airborne and orbital platforms.

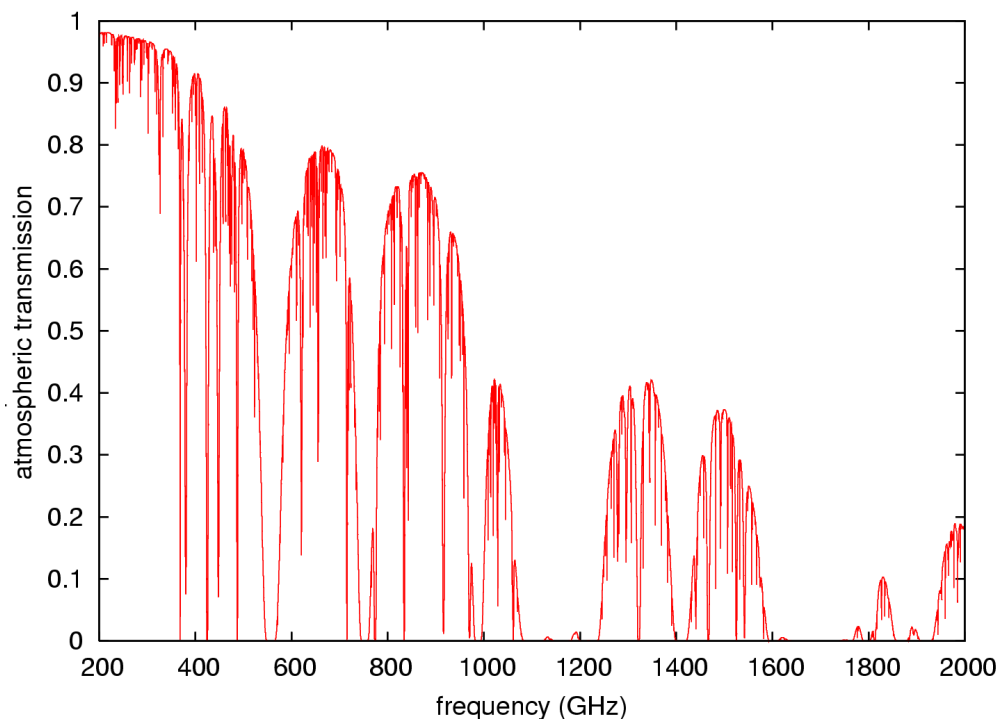

Figure 9: Preliminary model of 0.2 to $2 \mathrm{THz}$ atmospheric transmission over Dome A based on the current suite of winter measurements at $660 \mathrm{GHz}$ from Pre-HEAT.

The exceptional submillimeter capabilities of sites like Dome A and its counterpart in the highest peaks above the ALMA site in Chile represent great promise for enabling new science at submillimeter and terahertz frequencies for many years to come.

\section{ACKNOWLEDGEMENTS}

The authors wish to thank all members of the Polar Research Institute of China's 2008 Dome A expedition for their heroic efforts in reaching the summit and for providing invaluable assistance to the expedition astronomers in setting up the PLATO observatory and its attendant instruments. Construction of Pre-HEAT was financially supported from the US National Science Foundation under the auspices of Small Grants for Exploratory Research (ANT-0735854). 


\section{REFERENCES}

[1] Marrone, D. P., Blundell, R., Tong, E., Paine, S. N., Loudkov, D., Kawamura, J. H., Luhr, D., Barrientos, C., "Observations in the 1.3 and $1.5 \mathrm{THz}$ Atmospheric Windows with the Receiver Lab Telescope", 16th International Symposium on Space Terahertz Technology (2005).

[2] Kawamura, A., Mizuno, N., Yonekura, Y., Onishi, T., Mizuno, A., Fukui, Y., "NANTEN2: A Submillimeter Telescope for Large Scale Surveys at Atacama", Astrochemistry: Recent Successes and Current Challenges, Proceedings of the 231st Symposium of the International Astronomical Union, p 275 (2005).

[3] Ezawa, H., Kawabe, R., Kohno, K., Yamamoto, S., "The Atacama Submillimeter Telescope Experiment (ASTE)”, Proc. SPIE, 5489, 763-772 (2004).

[4] Güsten, R., et al., “APEX: the Atacama Pathfinder EXperiment”, Proc. SPIE, 6267, 37 (2006).

[5] Sebring, T.A., Giovanelli, R., Radford, S., Zmuidzinas, J., "Cornell Caltech Atacama Telescope (CCAT): a 25-m aperture telescope above 5000-m altitude", Proc. SPIE, 6267, 75 (2006).

[6] Chamberlin, R., "Comparisons of saturated water vapor column from radiosonde, and $\mathrm{mm}$ and submm radiometric opacities at the South Pole", ASP Conference Proceedings, 266, 172 (2002).

[7] Chamberlin, R.A., Martin, R.N., Martin, C.L., Stark, A.A., "Submillimeter atmospheric FTS at the geographic South Pole”, Proc. SPIE, 4855, 609-620 (2003).

[8] Oberst, T. E., Parshley, S. C., Stacey, G. J., Nikola, T., Löhr, A., Harnett, J. I., Tothill, N. F. H., Lane, A. P., Stark, A. A., Tucker, C. E., "Detection of the 205 um [N II] Line from the Carina Nebula", ApJ, 652, L125-L128, (2006).

[9] Lawrence, J. S., Ashley, M. C. B., Burton, M. G., Calisse, P. G., Dempsey, J. T., Everett, J. R., Mather, O., Storey, J. W. V., Travouillon, T., "The AASTINO: Automated Astrophysical Site Testing International Observatory", Proceedings of the Conference on Towards Other Earths: DARWIN/TPF and the Search for Extrasolar Terrestrial Planets, ESASP 539, 497-501 (2003).

[10] Calisse, P.G., Ashley, M.C. B., Burton, M.G., Phillips, M.A., Storey, J.W.V., Radford, S.J. E., Peterson, J.B., "Submillimeter Site Testing at Dome C, Antarctica", PASA, 21, 256-263 (2004).

[11] Lawrence, J. S., "Infrared and Submillimeter Atmospheric Characteristics of High Antarctic Plateau Sites”, PASP, 116, 482-492, (2004).

[12] Australian Antarctic Division, Australian Antarctic Data Centre website: http://www.aad.gov.au/weather/aws/domea/ , (2008).

[13] Lawrence, J.S. et al., "The PLATO Antarctic site testing observatory”, Proc. SPIE, these proceedings, (2008).

[14] Dame, T. M., Hartmann, Dap, Thaddeus, P., "The Milky Way in Molecular Clouds: A New Complete CO Survey”, ApJ, 547, 792-813 (2001).

[15] Siegel, P.H., Mehdi, I., Dengler, R.J., Lee, T.H., Humphrey, D.A., Pease, A., Zimmermann, R., Zimmermann, P., “A $640 \mathrm{GHz}$ planar-diode fundamental mixer/receiver”, Microwave Symposium Digest, 2, 407-410 (1998).

[16] Groppi, C. et al. "SuperCam: a 64 pixel heterodyne imaging spectrometer”, Proc. SPIE, these proceedings, (2008).

[17] Paine, S., “am atmospheric model”, SMA memo \#152, (2004). 